\title{
From Gas to Stars in Energetic Environments: Dense Gas Clumps in the 30 Doradus Region
}

\author{
C. N. Anderson ${ }^{1}$, D. S. Meier ${ }^{1}$, J. Ott ${ }^{2,1}$, A. Hughes ${ }^{3}$, T. Wong ${ }^{4}$ \\ ${ }^{1}$ Physics Department, New Mexico Tech, 801 Leroy Pl., Socorro, NM 87801, USA \\ email: canderso@nmt.edu, dmeier@nmt.edu \\ ${ }^{2}$ National Radio Astronomy Observatory, P.O. Box O, Socorro, NM 87801, USA \\ email: jott@nrao.edu \\ ${ }^{3}$ Max Plank Institute of Astronomy, Königstuhl 17 69117, Heidelberg, Germany \\ email: hughes@mpia-hd.mpg.de \\ ${ }^{4}$ Astronomy Department, University of Illinois, 1002 W. Green Street, Urbana, IL 61801, USA \\ email: wongt@illinois.edu
}

\begin{abstract}
We present parsec-scale interferometric maps of $\mathrm{HCN}(1-0)$ and $\mathrm{HCO}^{+}(1-0)$ emission from dense gas in the star-forming region 30Dor10, obtained using the Australia Telescope Compact Array. This extreme star-forming region, located in the Large Magellanic Cloud, is characterized by a very intense ionizing radiation field and sub-solar metallicity, both of which are expected to affect molecular cloud structure. We detect 13 clumps of dense molecular gas, some of which are aligned in a filamentary structure. Our analysis of the clump properties shows that they have similar mass but slightly wider linewidths than clumps detected in other LMC star-forming regions.
\end{abstract}

Keywords. ISM: molecules — Magellanic Clouds — galaxies: ISM

Figure 1 shows the first detection of resolved dense clumps in 30Dor10. Our results suggests that overall 30Dor10 is more turbulent, but possibly the dense cores represent a kinematically distinct and coherent filamentary structure. Also, we find that $\mathrm{HCO}^{+} / \mathrm{HCN}$ is low compared to Galactic GMCs and other (high metallicity) starbursts, and is at the low end of that observed in the LMC. This work is supported by the NSF grant AST1009620. CA's attendance at IAU292 was supported by an International Travel Grant from the AAS and the NSF.
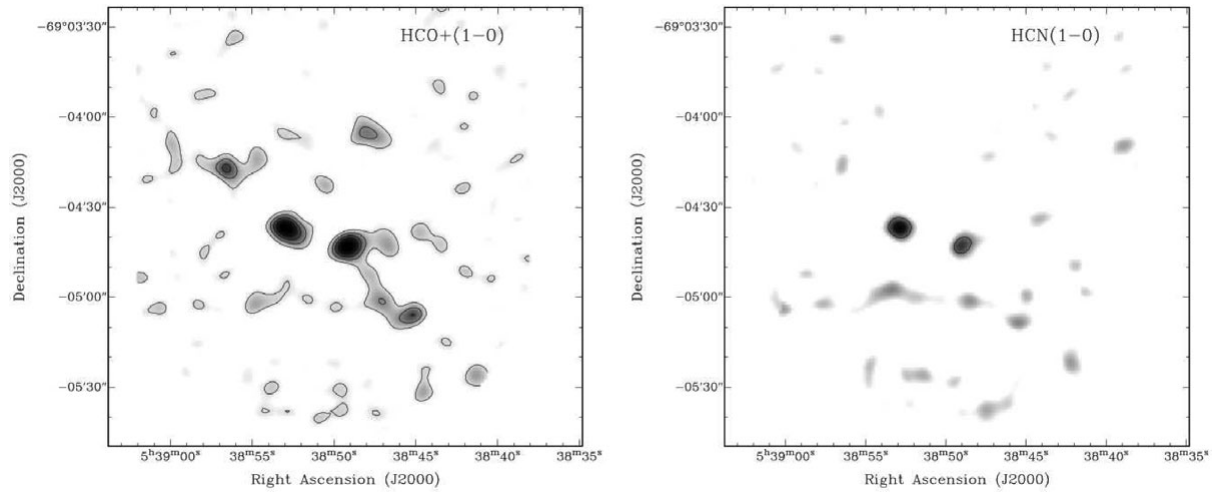

Figure 1. $\mathrm{HCO}^{+}$and $\mathrm{HCN}$ moment maps of 30Dor10 